\title{
Systems of Points with Coulomb Interactions
}

\author{
Sylvia Serfaty (New York University, USA)
}

Large ensembles of points with Coulomb interactions arise in various settings of condensed matter physics, classical and quantum mechanics, and even approximation theory, and give rise to a variety of questions pertaining to calculus of variations, partial differential equations and probability. We will review motivations from these fields as well as "mean-field limit" results that allow us to derive effective models and equations describing those systems at the macroscopic scale. We then explain how to analyse the next-order beyond the mean-field limit, obtaining information about systems at the microscopic level. In the setting of statistical mechanics, this allows, for instance, the observation of the effect of temperature and a connection with crystallisation questions.

\section{General setup}

The 18th century physicist Charles-Augustin de Coulomb was the first to postulate that electrically charged particles interact with one another by a force proportional to the inverse square of their distance apart, in a way similar to Newton's gravitational force. In this paper, we are interested in large systems of points (or particles) interacting by such forces, having as motivation, besides the case of classical mechanics, numerous other situations that we will detail below.

Recalling that force is the gradient of energy, we consider a system of $N$ particles with energy of the form

$$
\mathcal{H}_{N}\left(x_{1}, \ldots, x_{N}\right)=\frac{1}{2} \sum_{1 \leq i \neq j \leq N} \mathrm{~g}\left(x_{i}-x_{j}\right)+N \sum_{i=1}^{N} V\left(x_{i}\right) .
$$

Here, the points $x_{i}$ belong to the Euclidean space $\mathbb{R}^{\mathrm{d}}$, although it is also interesting to consider points on manifolds. The interaction kernel $\mathrm{g}(x)$ is taken to be

$$
\begin{aligned}
& g(x)=-\log |x|, \quad \text { in dimension } \mathrm{d}=2, \\
& \mathrm{~g}(x)=\frac{1}{|x|^{\mathrm{d}-2}}, \quad \text { in dimension } \mathrm{d} \geq 3 .
\end{aligned}
$$

Up to a multiplicative constant, this is the Coulomb kernel in dimension $d \geq 2$, i.e. the fundamental solution to the Laplace operator, solving

$$
-\Delta \mathrm{g}=\mathrm{c}_{\mathrm{d}} \delta_{0}
$$

where $\delta_{0}$ is the Dirac mass at the origin and $\mathrm{c}_{\mathrm{d}}$ is an explicit constant depending only on the dimension.

It is also interesting to broaden the study to the onedimensional logarithmic case

$$
\mathrm{g}(x)=-\log |x|, \quad \text { in dimension } \mathrm{d}=1,
$$

which is not Coulombian, and to more general Riesz interaction kernels of the form

$$
g(x)=\frac{1}{|x|^{\mathrm{s}}}, \quad \mathrm{s}>0 .
$$

The one-dimensional Coulomb interaction with kernel $-|x|$ is also of interest but has been extensively studied and is well understood.

We also include a possible external field or confining potential $V$, which is assumed to be sufficiently smooth and tending to infinity fast enough at infinity. The factor $N$ in front of $V$ makes the total confinement energy of the same order as the total repulsion energy, effectively balancing them and confining the system to a subset of $\mathbb{R}^{d}$ of fixed size.

The Coulomb interaction and the Laplace operator are obviously extremely important and ubiquitous in physics, as the fundamental interactions of nature (gravitation and electromagnetic) are Coulombic. Below, we will further review the reasons for studying this type of system.

There are several mathematical problems that are interesting to study, all in the asymptotic limit $N \rightarrow \infty$ :

(1) Understand the minimisers and possibly critical points of (1.1).

(2) Understand the statistical mechanics of systems with energy $\mathcal{H}_{N}$ and inverse temperature $\beta>0$, governed by the so-called Gibbs measure

$$
d \mathbb{P}_{N, \beta}\left(x_{1}, \ldots, x_{N}\right)=\frac{1}{Z_{N, \beta}} e^{-\beta \mathcal{H}_{N}\left(x_{1}, \ldots, x_{N}\right)} d x_{1} \ldots d x_{N} .
$$

Here, $\mathbb{P}_{N, \beta}$ is the probability density of observing the system in the configuration $\left(x_{1}, \ldots, x_{N}\right)$ if the inverse of the temperature is $1 / \beta$. The constant $Z_{N, \beta}$, which is called the "partition function" in physics, is the normalisation constant that makes $\mathbb{P}_{N, \beta}$ a probability measure, ${ }^{1}$ i.e.,

$$
Z_{N, \beta}=\int_{\left(\mathbb{R}^{\mathrm{d}}\right)^{N}} e^{-\beta \mathcal{H}_{N}\left(x_{1}, \ldots, x_{N}\right)} d x_{1} \ldots d x_{N} .
$$

(3) Understand the dynamic evolutions associated to (1.1), such as the gradient flow of $\mathcal{H}_{N}$ given by the system of coupled ODEs

$$
\dot{x}_{i}=-\frac{1}{N} \nabla_{i} \mathcal{H}_{N}\left(x_{1}, \ldots, x_{N}\right),
$$

the conservative dynamics given by the system of ODEs

$$
\dot{x}_{i}=\frac{1}{N} \mathbb{J} \nabla_{i} \mathcal{H}_{N}\left(x_{1}, \ldots, x_{N}\right)
$$

where $\mathbb{J}$ is an antisymmetric matrix, or the Hamiltonian dynamics given by Newton's law

$$
\ddot{x}_{i}=-\frac{1}{N} \nabla_{i} \mathcal{H}_{N}\left(x_{1}, \ldots, x_{N}\right) \text {. }
$$

We can also be interested in these dynamics with added noise.

\footnotetext{
1 One does not know how to explicitly compute the integrals (1.8) except in the particular case of (1.5) for specific cases of $V$ where they are called Selberg integrals (see [Fo]).
} 
From a mathematical point of view, the study of such systems touches on several fields of mathematical analysis (partial differential equations, calculus of variations, approximation theory), probability theory, mathematical physics and even geometry (when one considers such systems on manifolds). Some of the crystallisation questions they lead to also overlap with number theory, as we will see below.

\section{Motivations}

There is a large number of motivations for the study of the above questions. We briefly describe some of them:

(1) In superconductors, superfluids and Bose-Einstein condensates, one observes the occurrence of quantised "vortices", which behave mathematically like interacting particles with two-dimensional Coulomb interactions. In these systems, the vortices repel each other logarithmically, while being confined together by the effect of the magnetic field or rotation, and the result of the competition between these two effects is that, as predicted by Abrikosov, the vortices arrange themselves in a perfect triangular lattice pattern, called an Abrikosov lattice (see Figure 1; for more pictures, see www.fys.uio.no/super/ vortex/).

These systems are, in fact, described by an energy (the Ginzburg-Landau energy) and associated PDEs but we can show rigorously (in a study started by BethuelBrezis-Hélein and continued by [SS]; see also [Se1]) that, in the case (1.2), the analysis of the vortices is reduced to the discrete problems described above.

Another motivation is the analysis of vortices in classical fluids, such as that initiated by Onsager (see [MP]) or in plasma physics.

(2) Fekete points in approximation theory: these points arise in interpolation theory as the points minimising interpolation errors for numerical integration. They are defined as those points maximising the quantity

$$
\prod_{i \neq j}\left|x_{i}-x_{j}\right|
$$

or, equivalently, minimising

$$
-\sum_{i \neq j} \log \left|x_{i}-x_{j}\right|
$$

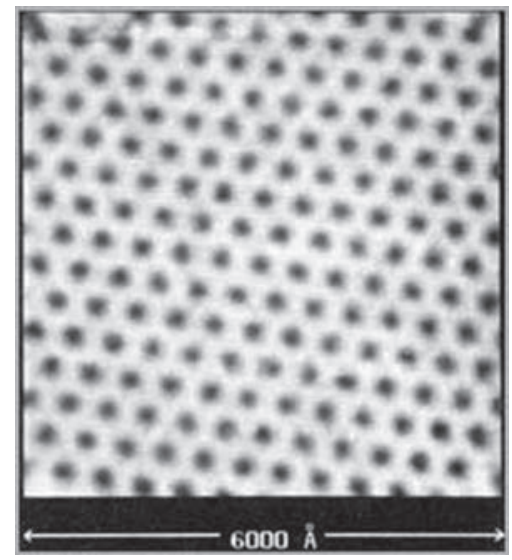

Figure 1. Vortices (in black) forming an Abrikosov lattice. H. F. Hess et al., Bell Labs, Phys. Rev. Lett. 62, 214 (1989).

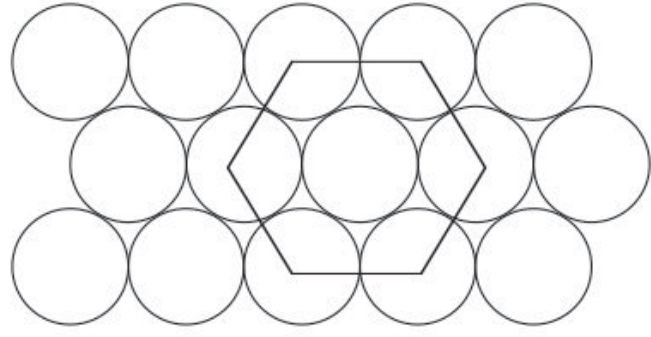

Figure 2. Solution of the sphere packing problem in dimension 2

They are often studied on the sphere or on other manifolds. In approximation theory [SK], we are also interested in the minimisation of Riesz energies

$$
\sum_{i \neq j} \frac{1}{\left|x_{i}-x_{j}\right|^{s}}
$$

for all values of $s$. One can show that, by letting $s \rightarrow 0$, the minimisers of Riesz energies converge to those of the logarithmic energy, whereas when $s \rightarrow \infty$, they converge to the minimisers of the optimal sphere packing problem (whose solution in dimension 2 is known, from Fejes Tóth, to be the triangular lattice represented in Figure 2). It has been proven by Hales that the solution of the same packing problem in dimension 3 is an FCC (face-centred cubic) lattice, as was conjectured by Kepler. In higher dimensions, the solution is only known in dimensions 8 and 24, due to a recent breakthrough by Viazovska (see the presentation in [Coh] and the review [S1]). In high dimensions, where the problem is important for error correcting codes, the solution is expected not to be a lattice.

(3) Statistical mechanics and quantum mechanics: in physics, the ensemble given by (1.7) in the Coulomb case is called a two-dimensional Coulomb gas or a one-component plasma and is a classic ensemble of statistical mechanics whose analysis is considered difficult due to the long range of the interactions. The study of the twodimensional Coulomb gas, as well as the one-dimensional $\log$ gas, is also motivated by the analysis of certain quantum wave-functions (fractional quantum Hall effect, free fermions in a magnetic field, etc.), as well as by several stochastic models in probability (see [Fo]). The variant of the two-dimensional Coulomb case with coexisting positive and negative charges is interesting in certain theoretical physics models (XY-model, sine-Gordon), which exhibit a Kosterlitz-Thouless phase transition (see [Spe]).

(4) Random matrices (see [Fo]): in the particular cases (1.5) and (1.2), the Gibbs measure (1.7) corresponds, in certain instances, to the distribution law of the eigenvalues of certain well known ensembles:

- The law of the complex eigenvalues of an $N \times N$ matrix where the entries are Gaussian i.i.d. is (1.7) with (1.2), $\beta=2$ and $V(x)=|x|^{2}$. This is called the Ginibre ensemble.

- The law of the real eigenvalues of an $N \times N$ Hermitian matrix with complex Gaussian i.i.d. entries is (1.7) with (1.5), $\beta=2$ and $V(x)=x^{2} / 2$. This is called the GUE (unitary Gaussian) ensemble.

- The law of the real eigenvalues of an $N \times N$ symmetric matrix with Gaussian i.i.d. entries is (1.7) with (1.5), 
$\beta=1$ and $V(x)=x^{2} / 2$. This is called the GOE (orthogonal Gaussian) ensemble.

(5) Complex geometry provides other examples of motivations. See, for instance, the works of Robert Berman and co-authors.

\section{The mean-field limit and theoretical physics}

\section{Questions}

The first question that naturally arises is to understand the limit as $N \rightarrow \infty$ of the empirical measure defined by

$$
\mu_{N}:=\frac{1}{N} \sum_{i=1}^{N} \delta_{x_{i}}
$$

for configurations of points that minimise the energy (1.1), critical points, solutions of the evolution problems presented above and typical configurations under the Gibbs measure (1.7), thus hoping to derive effective equations or minimisation problems that describe the average or mean-field behaviour of the system. The term mean-field refers to the fact that, from a physics point of view, each particle feels the collective (mean) field $\mathrm{g} * \mu_{N}$ generated by all the other particles. Convergence in the mean-field is thus equivalent, in some sense, to the "propagation of molecular chaos" (see [Go]). From the statistical mechanics point of view, we also try to understand the temperature dependence of the behaviour of the system and the eventual occurrence of phase transitions.

\section{The equilibrium measure}

The energy (1.1) can be written as

$$
\begin{aligned}
\mathcal{H}_{N}\left(x_{1}, \ldots, x_{N}\right) & \\
=N^{2}\left(\frac{1}{2} \iint_{\mathbb{R}^{\mathrm{d}} \times \mathbb{R}^{\mathrm{d}} \backslash \Delta} \mathrm{g}(x-y)\right. & d \mu_{N}(x) d \mu_{N}(y) \\
& \left.+\int_{\mathbb{R}^{\mathrm{d}}} V(x) d \mu_{N}(x)\right),
\end{aligned}
$$

where $\Delta$ denotes the diagonal of $\mathbb{R}^{d} \times \mathbb{R}^{d}$. Thus, it is natural to consider the "continuum version" of the energy, namely,

$$
\mathcal{I}_{V}(\mu):=\frac{1}{2} \iint_{\mathbb{R}^{d} \times \mathbb{R}^{d}} g(x-y) d \mu(x) d \mu(y)+\int_{\mathbb{R}^{d}} V(x) d \mu(x) .
$$

It is well known from potential theory that, in the space of probability measures, $\mathcal{I}_{V}$ admits a unique minimiser, $\mu_{V}$, which is called the equilibrium measure and is characterised by the fact that there exists a constant $c$ such that

$$
\left\{\begin{array}{cc}
h^{\mu_{V}}+V \geq c & \text { in } \mathbb{R}^{\mathrm{d}} \\
h^{\mu_{V}}+V=c & \text { in the support of } \mu_{V},
\end{array}\right.
$$

where

$$
h^{\mu_{V}}(x):=\int_{\mathbb{R}^{d}} \mathrm{~g}(x-y) d \mu_{V}(y)
$$

is the (electric) potential generated by $\mu_{V}$. This is true for Coulomb and for Riesz interactions, as well as for more general kernels. In the Coulomb case, the equilibrium measure can be interpreted with the help of an obstacle problem, and in the Riesz case with a fractional obstacle problem (see [Se1, Chap. 2]). An example is provided by Coulomb interaction (in any dimension) with confinement potential $V=c|x|^{2}$. In this case, we can verify that the equilibrium measure is always a (multiple of the) characteristic function of a ball. In the context of the Ginibre ensemble in Random Matrix Theory, this is known as the "circle law". Another important example is that of the logarithm interaction in dimension 1 with quadratic potential $V$ : the equilibrium measure has density $\sqrt{x^{2}-a^{2}} \mathbf{1}_{|x|<a}$, known in Random Matrix Theory as the (Wigner) semi-circle law for the ensembles GOE and GUE.

The energy $\mathcal{I}_{V}$ is the "mean-field limit" of the energy $\mathcal{H}_{N}$ and one can show, without much difficulty, that for the minimisers of $\mathcal{H}_{N}$, the empirical measure converges to $\mu_{V}$ and $\frac{1}{N^{2}} \min \mathcal{H}_{N}$ converges to $\mathcal{I}_{V}\left(\mu_{V}\right)$.

We can interpret $\nabla\left(h^{\mu}+V\right)$ as the total mean-force felt by a distribution with density $\mu$. Therefore, in view of (3.2), it is null for the minimisers. More generally, we expect that the critical points of $\mathcal{H}_{N}$ have a limiting empirical distribution satisfying

$$
\nabla\left(h^{\mu}+V\right) \mu=0 .
$$

For the dynamics (3), the formal limit of (1.9) or (1.10) is

$$
\partial_{t} \mu=-\operatorname{div}\left(\nabla\left(h^{\mu}+V\right) \mu\right)
$$

or

$$
\partial_{t} \mu=-\operatorname{div}\left(\mathbb{J} \nabla\left(h^{\mu}+V\right) \mu\right),
$$

again with $h^{\mu}=\mathrm{g} * \mu$. In the case (1.2), (3.6) with $V=0$ is also well known as the vorticity form of Euler's equation.

The difficulty in rigorously proving the convergence toward solutions of these equations (whose well-posedness also needs to be proved) consists of passing to the limit in products of the type $\nabla h^{\mu} \mu$, which are nonlinear and a priori ill-defined in energy space. In the case of (1.2), we can overcome these difficulties by a reformulation of the terms introduced by Delort in the context of his works in fluid mechanics but this approach does not work in higher dimensions.

Until recently, all convergence results were limited to subCoulomb singularities ( $s<d-2$ ) or to dimension 1 . Recently, a modulated energy method developed in [Se2] for the mean-field limit of the Ginzburg-Landau equations, based on the stability of solutions of the limiting equations for the "Coulomb norm" (or "Riesz norm")

$$
\|\mu\|^{2}=\iint \mathrm{g}(x-y) d \mu(x) d \mu(y),
$$

allowed for the treatment of Coulomb interactions and for more singular Riesz cases.

Theorem 1 ([Se3]). For the dynamics (1.9) and (1.10), for all $\mathrm{d}$, and all $\mathrm{s} \in[\mathrm{d}-2, \mathrm{~d})$ in (1.6), or (1.5) or (1.2), the empirical measures converge to the solutions of (3.5) or (3.6), when $N \rightarrow+\infty$, provided these are sufficiently smooth and the initial data energies converge to those of their limits.

This result was preceded by one by Duerinckx in dimension 1 and 2 for $\mathrm{s}<1$.

As far as (1.11) is concerned, the limiting equation is formally found to be the Vlasov-Poisson equation

$$
\partial_{t} \rho+v \cdot \nabla_{x} \rho+\nabla\left(h^{\mu}+V\right) \cdot \nabla_{v} \rho=0,
$$

where $\rho(t, x, v)$ is the density of particles at time $t$ with position $x$ and velocity $v$, and $\mu(t, x)=\int \rho(t, x, v) d v$ is the density of particles. Notwithstanding recent progress, we do not yet know how to prove convergence of (1.11) to (3.7) when the interaction is Coulomb or has a stronger singularity. About this topic, one can consult the reviews [Jab, Go]. 


\section{With temperature: statistical mechanics}

Let us now consider (1.8) and turn our attention to problem (2). It is known that even with temperature the behaviour of the system is still governed by the equilibrium measure. The result can be phrased using the language of Large Deviations Principles and states, essentially, that if $E$ is a subset of the space of probability measures, after identifying the configurations $\left(x_{1}, \ldots, x_{N}\right)$ in $\left(\mathbb{R}^{\mathrm{d}}\right)^{N}$ with their empirical measures, we have

$$
\mathbb{P}_{N, \beta}(E) \approx e^{-\beta N^{2}\left(\min _{E} \mathcal{I}_{V}-\min I_{V}\right)},
$$

which implies, due to the uniqueness of the minimiser $\mu_{V}$ of $\mathcal{I}_{V}$, that the configurations for which the empirical measure do not converge to $\mu_{V}$ have a very small probability. For example, in the case of matrices in GOE or GUE, for which the equilibrium measure is the semi-circle law, we deduce as an application a corollary of a result by Ben Arous and Guionnet: the probability that a GOE or GUE matrix is definite positive (and, thus, that all their eigenvalues are positive, which is incompatible with the semi-circle law because it is symmetric relative to 0 ) decreases like $e^{-c N^{2}}$.

In other words, at this leading order, temperature does not affect the mean-field behaviour of the system. (This is not what happens if we replace $\beta$ by $\beta / N$ : in this case, we have a modified equilibrium measure that spreads out with the temperature, minimising $\beta I_{V}(\mu)+\int \mu \log \mu$.)

\section{$4 \quad$ Beyond mean-field}

In order to observe, for example, the effect of temperature (see Figure 3), it is interesting to go beyond the mean-field limit: expanding the energy $\mathcal{H}_{N}$ to next order we have, at the same time, access to information about the typical microscopic behaviour of the configurations. Observe that, at the microscopic scale, the typical distance between nearest neighbours is $N^{-1 / \mathrm{d}}$.

\section{Rigidity and Gaussian fluctuations}

For minimisers of the energy $\mathcal{H}_{N}$ or of typical configurations under (1.7), since one already knows that $\sum_{i=1}^{N} \delta_{x_{i}}-N \mu_{V}$ is small, one knows, for instance, that the so-called discrepancy in balls $B_{r}(x)$, defined by

$$
D(x, r):=\int_{B_{r}(x)} \sum_{i=1}^{N} \delta_{x_{i}}-N d \mu_{V},
$$

is of order $o\left(r^{\mathrm{d}} N\right)$ for fixed $r>0$. It can be asked whether this estimation can be refined and if it remains true at mesoscopic

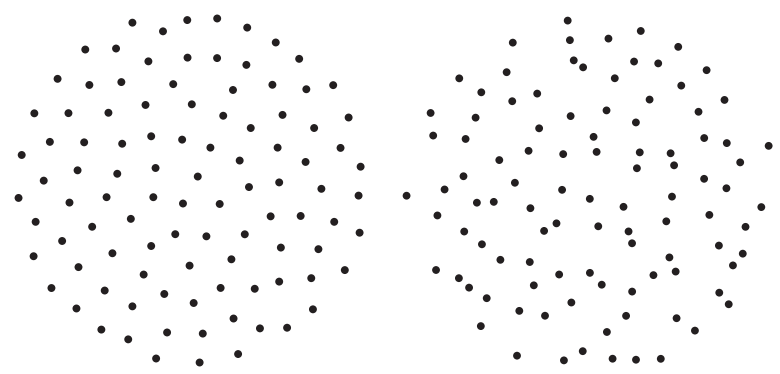

Figure 3. Case (1.2) with $N=100$ and $V(x)=|x|^{2}$, for $\beta=400$ (left) and $\beta=5$ (right) scales, i.e., for $r$ of order $N^{-\alpha}$ with $\alpha<1 / \mathrm{d}$, and for all temperatures. This would correspond to a rigidity result. We do get such a result for the energy minimisers. For configurations with temperature, in the context of bi-dimensional Coulomb interactions, we can prove a slightly different form of such a result: it is true when we integrate $\sum_{i=1}^{N} \delta_{x_{i}}-N \mu_{V}$ not over a ball but against a sufficiently smooth test function. In this way, we get an even more precise result, since we can prove that these quantities converge to a Gaussian with explicitly known mean and variance.

Theorem 2 ([LS2]). In case (1.2), let us assume that $V \in C^{4}$ and $\mu_{V}$ has connected support $\Sigma$ with a regular boundary. Let $f \in C_{c}^{3}(\Sigma)$. Then,

$$
\sum_{i=1}^{N} f\left(x_{i}\right)-N \int_{\Sigma} f d \mu_{V}
$$

converges in law to a Gaussian with

$$
\begin{aligned}
& \text { mean }=\frac{1}{2 \pi}\left(\frac{1}{\beta}-\frac{1}{4}\right) \int_{\mathbb{R}^{2}} \Delta f \log \Delta V, \\
& \text { variance }=\frac{1}{2 \pi \beta} \int_{\mathbb{R}^{2}}|\nabla f|^{2} .
\end{aligned}
$$

This result can be localised with test-functions $f$ supported on any mesoscale $N^{-\alpha}, \alpha<\frac{1}{2}$. It is also true for energy minimisers, formally taking $\beta=\infty$.

For an idea of the proof, we suggest the lecture notes [Se4].

This result can be interpreted in terms of the convergence to a suitable Gaussian free field, a sort of two-dimensional analogue of Brownian motion. Note that a similar result was obtained by Bauerschmidt-Bourgade-Nikula-Yau and it was previously known for $\beta=2$, and in the uni-dimensional logarithm case for all values of $\beta$.

If $f$ is sufficiently smooth, the associated fluctuations are typically of order 1, i.e., much smaller than we could expect, for example comparing with the standard Central Limit Theorem, where the fluctuation of the sum of $N$ i.i.d. random variables is typically of order $\sqrt{N}$. Proving this result in higher dimension or for more general interactions remains an open problem.

\section{Next order in the energy}

As we pointed out above, the approach we employ (initiated with Etienne Sandier and continued with Nicolas Rougerie, Mircea Petrache and Thomas Leblé) consists of studying the next order of the expansion of the energy about the measure $N \mu_{V}$, which is formally the minimiser. Expanding and using the characterisation (3.2), the "order 1 " terms in $\sum_{i=1}^{N} \delta_{x_{i}}$ $N \mu_{V}$ vanish and we obtain

$$
\mathcal{H}_{N}\left(x_{1}, \ldots, x_{N}\right)=N^{2} \mathcal{I}_{V}\left(\mu_{V}\right)+F_{N}^{\mu_{V}}\left(x_{1}, \ldots, x_{N}\right),
$$

where

$$
\begin{aligned}
& F_{N}^{\mu_{V}}\left(x_{1}, \ldots, x_{N}\right) \\
= & \frac{1}{2} \iint_{\Delta^{c}} \mathrm{~g}(x-y) d\left(\sum_{i=1}^{N} \delta_{x_{i}}-N \mu_{V}\right)(x) d\left(\sum_{i=1}^{N} \delta_{x_{i}}-N \mu_{V}\right)(y)
\end{aligned}
$$


and again $\triangle$ denotes the diagonal $\mathbb{R}^{\mathrm{d}} \times \mathbb{R}^{\mathrm{d}}$. This is a next-order expansion of $\mathcal{H}_{N}$ valid for arbitrary configurations.

The "next-order energy" $F_{N}^{\mu_{V}}$ can be seen as the total Coulomb energy of the neutral system formed by $N$ positive point charges at points $x_{i}$ and a diffuse negative charge $-N \mu_{V}$ with the same mass. The goal is now to define the limit of this energy when $N \rightarrow \infty$, which will be the total Coulomb energy (per unit volume) of an infinite system of positive charges and a (let us say) uniformly distributed negative charge. In physics, such a system is called a jellium. The precise definition of this limiting energy is a bit complex but it uses, in a crucial way, the Coulomb nature of the interaction. In fact, since $\mathrm{g}$ is the kernel of the Laplacian, we observe that if $h^{\mu}=\mathrm{g} * \mu$ is the electrostatic potential generated by a charge distribution $\mu$ (with zero integral) then $h^{\mu}$ solves the Poisson equation

$$
-\Delta h^{\mu}=\mathrm{c}_{\mathrm{d}} \mu
$$

which is a local elliptic PDE. Additionally, using the GaussGreen formula, we can write

$$
\begin{aligned}
\iint_{\mathbb{R}^{\mathrm{d}} \times \mathbb{R}^{\mathrm{d}}} \mathrm{g}(x-y) d \mu(x) d \mu(y) & =-\frac{1}{\mathrm{C}_{\mathrm{d}}} \int_{\mathbb{R}^{\mathrm{d}}} h^{\mu} \Delta h^{\mu} \\
& =\frac{1}{\mathrm{C}_{\mathrm{d}}} \int_{\mathbb{R}^{\mathrm{d}}}\left|\nabla h^{\mu}\right|^{2} .
\end{aligned}
$$

In another way, we can rewrite the interaction energy (which involves a double integral) in the form of a single integral of a local function of the electrostatic (or Coulomb) potential generated by this distribution, itself a solution of a local equation. In Riesz's case, these manipulations can be replaced by similar ones using the fact that $g$ is the kernel of an elliptic operator in divergence form, which is still local.

With the help of this observation, we succeed in defining an infinite volume energy for an infinite configuration of points $C$ neutralised by a distributed charge (let us say -1 ) via the solutions of

$$
-\Delta H=\mathrm{c}_{\mathrm{d}}\left(\sum_{p \in C} \delta_{p}-1\right) .
$$

We shall denote this energy by $\mathbb{W}(C)$. When the configuration of points $C$ is periodic with respect to a lattice $\Lambda$, the energy $\mathbb{W}(C)$ has an explicit form: if there are $M$ points $a_{i}$ in the fundamental cell, we have (up to constants)

$$
\mathbb{W}(C)=\sum_{1 \leq i \neq j \leq M} G_{\mathbb{T}}\left(a_{i}, a_{j}\right)
$$

where $G_{\mathbb{T}}$ is the Green function of the torus $\mathbb{T}:=\mathbb{R}^{\mathrm{d}} / \Lambda$.

We can show that $\mathbb{W}$ can be obtained as the limit (in a certain sense) of the functional $F_{N}^{\mu_{V}}$ in (4.1). It also follows from an expansion to the next order of the minimum of the energy $\mathcal{H}_{N}$ and from the fact that, after dilation, the minimisers of $\mathcal{H}_{N}$ must converge (almost everywhere with respect to the origin of the dilation) to a minimiser of $\mathbb{W}$ (see, for example, $[\mathrm{Se} 1])$.

We are therefore led to try to determine the minimisers of $\mathbb{W}$. This problem is extremely difficult, with the exception of the one-dimensional case, where we can prove that the minimum of $\mathbb{W}$ is attained by the lattice $\mathbb{Z}$. In dimension 2 and higher, the problem remains open and the only positive result is the following.
Theorem 3. The minimum of $\mathbb{W}$ over lattices of volume 1 in dimension 2 is achieved uniquely by the triangular lattice.

Here, the triangular lattice means $\mathbb{Z}+\mathbb{Z} e^{i \pi / 3}$ properly scaled, i.e., what is called the Abrikosov lattice in the context of superconductivity. This partial result is, in fact, a result from number theory, known since the 1950s, about the minimisation of Epstein's zeta function (see [Mont] and references therein). It corresponds to minimising the height of a flat torus in Arakelov geometry.

Since the triangular lattice is observed in experiments with superconductors and since we have proved that the minimisation of the Ginzburg-Landau energy of the superconductor reduces to that of $\mathbb{W}[S S]$, it is natural to conjecture that the triangular lattice is a global minimiser of the energy.

According to a conjecture of Cohn-Kumar, the triangular lattice should be a universal minimizer in dimension 2 (i.e., should minimise a large class of interaction energies). An analogous role is played in dimension 8 by the lattice $E_{8}$ and in dimension 24 by the Leech lattice, which are solutions of the optimal packing problem, as was recently proved [Coh]. In these dimensions, the proof of the universal minimisation is near at hand.

In dimensions $d \geq 3$ (except for $d=8$ and $d=24$ ), the minimisation of $\mathbb{W}$, even among lattices, is an open problem. As before, we can think that this relative minimum is global but we expect this to be true only in low dimensions since computer simulations provide clear indications that in dimensions $d \geq 9$ the minimisers are not lattices.

These questions belong to the more general family of crystallisation problems for which very few positive results are known once the dimension is larger than or equal to 2 (see the review [BLe]).

\section{Next order with temperature}

In order to observe interesting temperature effects, as well as for applications to random matrices, we must consider $\beta_{N}=$ $\beta N^{\frac{2}{d}-1}$.

As we saw above, the macroscopic (or "mean-field") behaviour of the system does not depend upon the temperature and is given by the equilibrium measure. On the other hand, one can show that the microscopic behaviour depends on the temperature and is governed by a weighted sum of the energy $\mathbb{W}$ (from the previous paragraph) and a relative entropy. To formulate the result, one needs to dilate the configurations by $N^{-1 / \mathrm{d}}$, as in the previous paragraph, and consider the limiting point process $P^{x}$ obtained by averaging near each point $x$. Here, a point process is a law on infinite configurations of points. For instance, the Ginibre process is obtained by passing to the limit $N \rightarrow \infty$ (after dilation) of the Ginibre ensemble; the Poisson process $\Pi$ with intensity 1 corresponds to points thrown independently of each other in such a way that the probability of having $N(B)$ points in a set $B$ is

$$
\Pi(N(B)=n)=\frac{|B|^{n}}{n !} e^{-|B|} .
$$

Thus, one defines a "specific relative entropy" with respect to the Poisson process, denoted by ent[-|П], that we can think of as measuring how close the process $P$ is to Poisson. 

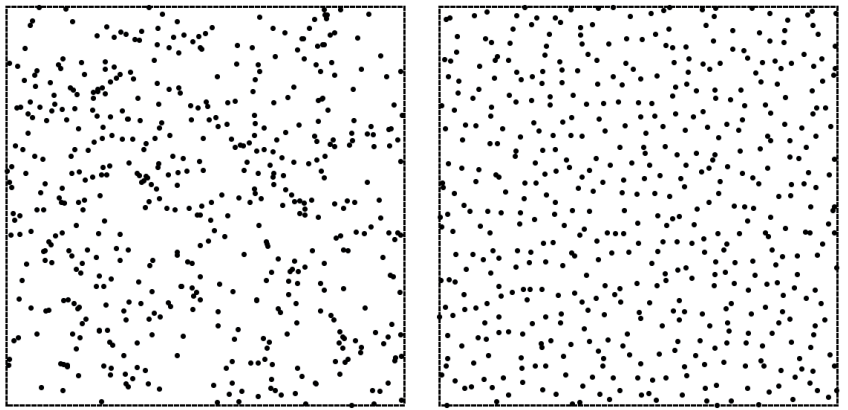

Figure 4. Simulation of the Poisson point process with intensity 1 (left) and the Ginibre process (right)

For all $\beta>0$, we define the functional $\mathcal{F}_{\beta}$

$$
\mathcal{F}_{\beta}(P):=\int_{\Sigma} \frac{\beta}{2} \mathbb{W}\left(P^{x}\right)+\operatorname{ent}\left[P^{x} \mid \Pi\right] d x
$$

with $P=\int_{\Sigma} P^{x} d x$. We can now formulate a large deviations result.

Theorem 4 ([LS1]). For all cases (1.5), (1.2) and (1.3) with $\mathrm{d}-2 \leq \mathrm{s}<\mathrm{d}$, with smooth assumptions on $V$ and $\mu_{V}$, and for all $\beta>0$, we have a Large Deviations Principle at speed $N$ with rate function $\mathcal{F}_{\beta}-\inf \mathcal{F}_{\beta}$, in the sense that

$$
\mathbb{P}_{N, \beta}\left(P_{N} \simeq P\right) \simeq e^{-N\left(\mathcal{F}_{\beta}(P)-\inf \mathcal{F}_{\beta}\right)}
$$

In this way, the Gibbs measure $\mathbb{P}_{N, \beta}$ concentrates on microscopic point processes that minimise $\mathcal{F}_{\beta}$. This minimisation is due to a competition between energy and entropy. When $\beta \rightarrow 0$, the entropy dominates and we can prove that the limit processes converge to a Poisson process. When $\beta \rightarrow \infty$, the energy $\mathbb{W}$ dominates, which, heuristically, forces the configurations to be more "ordered" and to converge to the minimisers of $\mathbb{W}$. Between these two extremes, we have intermediate situations and to know if there is a critical $\beta$ corresponding to a crystallisation, or to a liquid-solid phase transition (which is conjectured to take place for (1.2) in some physics papers), is a problem that remains open. In dimension 1 , on the other hand, due to the fact that we can identify the minimisers of $\mathbb{W}$, it can be concluded that a true crystallisation result holds when the temperature tends to 0 .

One consequence of this result is to provide a variational interpretation for the few known limiting processes: the socalled "sine- $\beta$ " process, the limit in the uni-dimensional case (1.5) and Ginibre's process, i.e., they must minimise $\beta \mathbb{W}+$ ent.

We would like to obtain more information about the limiting point processes, namely, the behaviour and decay of the "two-points correlation functions", which would shed light on the existence of phase transitions and crystallisation. Unfortunately, this theorem does not seem to provide much help for these problems.

As we have seen, many questions remain open, notably those of crystallisation, identification of minimisers and minima of $\mathbb{W}$ and $\mathcal{F}_{\beta}$, and the generalisation of Theorem 2 to dimensions $d \geq 3$, to Riesz interactions and even to more general interactions.

\section{References}

[BLe] X. Blanc, M. Lewin, The crystallization conjecture: A review. EMS surveys 2 (2015), 255-306.

[Coh] H. Cohn, A conceptual breakthrough in sphere packing. Notices AMS 64 (2017), no. 2, 102-115.

[Fo] P. J. Forrester, Log-Gases and Random Matrices. London Mathematical Society Monographs Series, 34. Princeton University Press, 2010.

[Go] F. Golse, On the Dynamics of Large Particle Systems in the Mean Field Limit. In Macroscopic and large scale phenomena: Coarse graining, mean field limits and ergodicity. Lect. Notes Appl. Math. Mech. 3 (2016), 1-144.

[Jab] P. E. Jabin, A review of the mean field limits for Vlasov equations. Kinet. Relat. Models 7 (2014), 661-711.

[LS1] T. Leblé, S. Serfaty, Large deviation principle for empirical fields of Log and Riesz gases. Invent. Math. 210, No. 3, 645-757.

[LS2] T. Leblé, S. Serfaty, Fluctuations of two-dimensional Coulomb gases. Geom. Funct. Anal. (GAFA), 28, no. 2, (2018), 443-508.

[MP] C. Marchioro, M. Pulvirenti, Mathematical Theory of Incompressible Nonviscous Fluids. Applied Mathematical Sciences, 96. Springer-Verlag, New York, 1994.

[Mont] H. L. Montgomery, Minimal theta functions. Glasgow Math J. 30, (1988), No. 1, 75-85, (1988).

[SK] E. Saff, A. Kuijlaars, Distributing many points on a sphere. Math. Intelligencer 19 (1997), no. 1, 5-11.

[SS] E. Sandier, S. Serfaty, From the Ginzburg-Landau model to vortex lattice problems. Comm. Math. Phys. 313 (2012), 635-743.

[Se1] S. Serfaty, Coulomb Gases and Ginzburg-landau Vortices. Zurich Lectures in Advanced Mathematics, 70, Eur. Math Soc., 2015.

[Se2] S. Serfaty, Mean field limits for the Gross-Pitaevskii and parabolic Ginzburg-Landau equations. J. Amer. Math. Soc. 30 (2017), no. 3, 713-768.

[Se3] S. Serfaty, Mean field limit for Coulomb flows, arXiv:1803.08345.

[Se4] S. Serfaty, Microscopic description of Log and Coulomb gases. Lecture notes of the PCMI, arXiv:1709.04089.

[S1] N. J. A. Sloane, The sphere packing problem, Proceedings Internat. Congress Math. Berlin 1998, Documenta Mathematika, III (1998), 387-396.

[Spe] T. Spencer, Scaling, the free field and statistical mechanics. In: The Legacy of Norbert Wiener: A. Centennial Symposium. Proc. Sympos. Pure Math 60, AMS (1997).

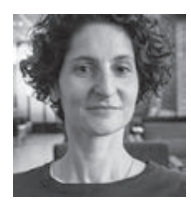

Sylvia Serfaty [serfaty@cims.nyu.edu] is interested in developing analysis tools to understand problems from physics. She has extensively studied the Ginzburg-Landau model of super-conductivity and this has led her to questions on the statistical mechanics of Coulomb-type systems. She has been splitting her career between Paris and New York.

This is an English translation by Fernando Pestana da Costa of the French article "Systèmes de points en interaction coulombienne" by Sylvia Serfaty, published in La Gazette des Mathématiciens (No. 157, pp. 29-37, Juillet 2018). The EMS Newsletter is thankful to the author, to La Gazette des Mathématiciens and to the Société Mathématique de France for authorisation to republish this article. 